\title{
LA EVOLUCIÓN DEL ANÁLISIS DE POLÍTICAS EN ESTADOS UNIDOS: CUATRO FUENTES DE DEMANDA ${ }^{1}$
}

DAvid L. Weimer

\section{INTRODUCGIÓN}

UNA DEFINICIÓN ESTRICTA DE ANÁLISIS de políticas como actividad profesional enfatiza la orientación hacia el cliente y la perspectiva pública. Es el caso de la siguiente definición: “análisis de políticas son consejos orientados al cliente, relevantes para las decisiones públicas y fundados en valores sociales". ${ }^{2}$ No obstante, una valoración integral del papel que desempeña el análisis de políticas en Estados Unidos requiere una definición más vasta, que incluya la investigación en políticas al relajar el criterio de que el análisis sea para un cliente físico o institucional. De esta forma, para los propósitos de esta revisión, defino el análisis de políticas como consejos emitidos por profesionales, relevantes para decisiones públicas y fundados en valores sociales. Además de empleados públicos que ocupan puestos de trabajo específicamente llamados "analistas de políticas”, incluyo a otros funcionarios públicos que consagran parte de su tiempo laboral al análisis de políticas, así como a personas en los sectores con y sin fines de lucro cuyas actividades profesionales implican dar consejos analíticos sobre políticas. De acuerdo a esta definición, una gran cantidad de personas en Estados Unidos

${ }^{1}$ Este ensayo fue preparado para una presentación en el Centro de Estudios Internacionales de El Colegio de México. Agradezco a Angela Evans por sus útiles comentarios.

${ }^{2}$ David L. Weimer y Aidan R. Vining, Policy Analysis: Concepts and Practice, $5^{\mathrm{a}}$ ed., Pearson, Upper Saddle River, NJ, 2011, p. 24. 
trabajan como analistas de políticas. ¿Qué factores han llevado a que el análisis de políticas sea una profesión común en la vida pública estadounidense?

En este ensayo identifico las que son, en mi consideración, las cuatro fuentes principales de demanda para el análisis de políticas en Estados Unidos: primero, como apoyo basado en evidencia para reformas (o promoción) de políticas; segundo, como una fuente de conocimiento especializado para respaldar decisiones legislativas y ejecutivas; tercero, como una respuesta al creciente alcance y complejidad en la interacción entre gobierno y sociedad; y cuarto, como un mecanismo para que los políticos se frenen a sí mismos y a otros de promover iniciativas que, aunque sean atractivas políticamente, no promueven el interés público. Discuto brevemente las raíces históricas de cada una de estas fuentes de demanda y esbozo su impacto en las prácticas contemporáneas. Termino subrayando los incentivos que han generado estas cuatro fuentes de demanda para que la educación superior provea egresados con aptitudes relevantes en el campo del análisis de políticas.

\section{DEMANDA SURGIDA POR LA VOLUNTAD DE REFORMAR Y PROMOVER POLÍTICAS}

En Estados Unidos hay una plétora de organizaciones sin fines de lucro cuya misión es proporcionar evidencia en áreas específicas de política o en apoyo a distintas "reformas" gubernamentales. Como la reforma deseada por una persona puede ser el mayor miedo de otra, quizás "promoción” [advocacy] sea un mejor término que "reforma". Aún así, creo que "reforma" es el término adecuado para las primeras organizaciones de este tipo, los Burós Municipales de Investigación (Municipal Research Bureaus), primeros grupos de reflexión, think tanks, estadounidenses. Aunque la palabra "reformar" describe adecuadamente los objetivos de los think tanks contemporáneos, la creciente diferenciación ideológica provoca conflictos entre alternativas de reforma en competencia.

$\mathrm{Al}$ inicio del siglo xx, el Movimiento Progresista había logrado un número importante de victorias electorales en grandes ciudades 
de Estados Unidos. No obstante, estas victorias eran por lo general revertidas en elecciones siguientes por maquinarias políticas locales y, por lo tanto, no impactaban a largo plazo en reducir la corrupción y aumentar la eficiencia de los gobiernos municipales. Un enfoque alternativo, propuesto por reformadores progresistas y financiado por ricos filántropos, fue la creación de organizaciones para juntar datos sobre servicios municipales y proponer cambios en la gestión de estos servicios que la hicieran más eficiente y menos propensa a la corrupción.

La primera de dichas organizaciones, financiada por Andrew Carnegie y John D. Rockefeller, entre otros, se fundó en 1906 en Nueva York. ${ }^{3}$ Esta organización se convertiría en el modelo para organizaciones similares en otras ciudades. ${ }^{4}$ En diez años, este modelo se había expandido a veintiún ciudades importantes, con la creación de organizaciones como El Buró Ciudadano (Citizen's Bureau) en Milwaukee, la Federación Cívica (Civic Federation) en Chicago, y la Comisión Encuestadora (Survey Commission) en Atlantic City. ${ }^{5}$ Una herramienta principal de los burós era la recolección sistemática de datos sobre la operación y organización de los gobiernos de la ciudad mediante "encuestas", que han sido descritas como la base empírica de la disciplina que emergió en administración pública. ${ }^{6}$ Estas encuestas eran extensas. Por ejemplo, el recién creado Buró para la Investigación Municipal de Rochester, financiado por George Eastman y otros filántropos locales, comisionó al Buró para la Investigación Municipal de Nueva York (New York Bureau of Municipal Research) implementando una encuesta sobre el gobierno de la ciudad de Rochester. El

${ }^{3}$ Hindy Lauer Schacter, "Democracy, Scientific Management and Urban Reform the Case of the Bureau of Municipal Research and the 1912 New York City School Inquiry", Journal of Management History, vol. 1, núm. 2, 1995, pp. 52-64.

${ }^{4}$ Norman N. Gill, Municipal Research Bureaus: A Study of the Nation's CitizenSupported Agencies, Washington, D. C., American Council on Public Affairs, 1944. También: Schacter, "Philadelphia's Progressive-Era Bureau of Municipal Research", Administrative Theory Ė Praxis, vol. 24, núm. 3, 2002, pp. 555-570.

${ }^{5}$ Gill, op. cit.

${ }^{6}$ Anthony M. Bertelli y Laurence E. Lynn, Jr., Madison's Managers: Public Administration and the Constitution, Baltimore, The Johns Hopkins University Press, 2006. 
reporte final, de 546 páginas, contenía una enorme cantidad de datos sobre la gestión del municipio y recomendaciones para apoyar los objetivos de la organización: "1. Lograr cambios en la comunidad, mediante la cooperación con funcionarios electos, para aumentar la eficiencia y reducir el desperdicio; 2. Fungir como una agencia independiente no partidista cuyo objetivo sea informar a los ciudadanos de los asuntos de la ciudad" (New York Municipal Bureau, 1915).

Los burós defendían la eficiencia, pero negaban influir en la política de gran alcance; anunciando la retórica contemporánea sobre la formulación de políticas basada en evidencia, decían proveer datos neutrales y consejos para mejorar la eficiencia en la $a d-$ ministración de la política. No obstante, dar consejos sobre cómo reorganizar y dotar de recursos humanos a los gobiernos municipales claramente concuerda con la definición de análisis de políticas. Además, "aunque el impulso a los burós se basa en la importancia de recopilar datos, los burós no deben ser considerados meras máquinas de pensamiento. Como dijo el director de la agencia de Detroit: 'Los burós piensan, sueñan, consultan, estimulan, educan, irritan y, si son buenos, cambian el enfoque gubernamental y la conciencia pública de la ciudad"."7

Además de la propagación de burós municipales privados, algunas ciudades crearon sus propias unidades, usualmente llamadas "burós para la eficiencia", para investigar la gestión urbana. ${ }^{8}$ Uno de los primeros ejemplos fue una agencia de investigación creada en Milwaukee por la administración socialista en $1910 .^{9}$ Los "burós para la eficiencia" realizaban estudios similares a las encuestas hechas por los burós municipales, y varias se convirtieron en oficinas municipales que en la actualidad aconsejan a los funcionarios sobre presupuesto y otros asuntos fiscales.

Más allá de temas de gestión en ciudades específicas, diversos think tanks, ahora comúnmente definidos como organizaciones de

${ }^{7}$ Gill, op. cit., p. 37.

${ }^{8}$ Mordecai Lee, Bureaus of Efficiency: Reforming Local Government in the Progressive Era, Milwaukee, Marquette University, 2008.

${ }^{9}$ Gill, op. cit. 
investigación, análisis y compromiso en políticas públicas, ${ }^{10}$ se crearon para proveer investigación a nivel de la política nacional. En 1916, la Institución Brookings (Brookings Institution) comenzó a proveer investigación basada en evidencia relevante para la política nacional. Acto seguido se crearon varios think tanks, incluidos el Buró Nacional de Investigación Económica (National Bureau of Economic Research) en 1920, el Instituto de Políticas Públicas American Enterprise (American Enterprise Institute for Publicy Policy) en 1938, la organización Recursos para el Futuro (Resources for the Future) en 1952, y el Instituto Urbano (Urban Institute) en 1968. El crecimiento de los think tanks en Estados Unidos se ha acelerado en los últimos veinte años: ahora superan los 1 800, de los cuales 550 están en o cerca de Washington, D. C. ${ }^{11}$

Aunque los primeros think tanks eran usualmente creados para defender algún tipo de reforma, hasta la década de los años ochenta la mayoría de los think tanks prominentes podían definirse como universidades sin estudiantes o como organizaciones de investigación por contrato, con el primer tipo proveyendo más investigación de políticas y el segundo más análisis de políticas (de acuerdo a la definición estricta). No obstante, desde entonces un tercer tipo de think tank, el "think tank promotor" [advocacy tank], o de cabildeo, ha proliferado. "Los tanks promotores combinan una línea política dura, un sesgo ideológico o partidista, un sistema de ventas agresivo y un esfuerzo por influir en los debates sobre las políticas en curso. Además, los think tanks promotores sintetizan y dan un "giro" particular a investigación existente, más que producir investigación original. Lo que puede faltar en términos de rigor académico se compensa por su accesibilidad para quienes toman las decisiones". ${ }^{12}$ Su posicionamiento tiende a seguir líneas ideológicas claras: ${ }^{13}$ conservadores (por ejemplo la Heritage Foundation, el American Enterprise

${ }^{10}$ James G. McGann, Think Tanks and Policy Advice in the US: Think Tanks and Civil Societies Program, Filadelfia, University of Pennsylvania, 2005.

11 Think Tanks and Civil Society Program, 2013 Global Go To Think Tank Index Report, Filadelfia, University of Pennsylvania, 22 de enero de 2014, pp. 21-23.

${ }^{12}$ R. Kent Weaver, “The Changing World of Think Tanks”, PS: Political Science E Politics, vol. 22, núm. 3, 1989, p. 567.

${ }^{13}$ McGann, op. cit., pp. 11-13. 
Institute, o el Manhattan Institute), liberal (Cato Institute, Reason Foundation), centro-derecha (Center for Strategic and International Studies), centro (Resources for the Future, National Bureau of Economic Research, Public Policy Institute of California), centro-izquierda (Urban Institute, Brookings Institution), izquierda (Center on Budget and Policy Priorities, Center for American Progress).

El aumento en el número y en la prominencia pública de estos think tanks tiene implicaciones positivas y negativas para el análisis de políticas. En el lado positivo de la balanza, estas organizaciones pueden proveer vetas de análisis de políticas más relevantes para los asuntos públicos que la investigación académica, usualmente guiada por el requisito disciplinario de la generalización más que por su relevancia para las decisiones de política pública. Además, el número elevado de think tanks permite que analistas de políticas se desempeñen profesionalmente fuera de las restricciones que significan trabajar en el gobierno. Los incentivos para agradar a donantes y obtener cobertura mediática hacen que estos think tanks traduzcan investigaciones en información directamente relevante para decisiones de política, y la diseminen intensamente. En el lado negativo de la balanza, una marcada orientación ideológica y una falta de disciplina en la investigación pueden mermar la credibilidad del análisis y conducir a una pelea por publicar estudios, en detrimento de la búsqueda por las mejores respuestas a preguntas cruciales de política. La preocupación por la respuesta de donadores potenciales puede también afectar la agenda de los think tanks. Se ha sugerido incluso que el predominio de los think tanks en "el cruce entre las esferas académicas, políticas, económicas y mediáticas" ha "socavado el valor del conocimiento producido de manera independiente [...] al institucionalizar un modo de práctica intelectual que relega a sus productores a los márgenes de la vida pública y política". ${ }^{14}$ Sea como uno incline la balanza, los think tanks desempeñan sin duda un papel vital en el análisis de políticas contemporáneo en Estados Unidos.

14 Thomas Medvetz, Think Tanks in America, Chicago, University of Chicago Press, 2012, p. 7. 


\section{LA DEMANDA SURGIDA POR LA NECESIDAD DE CONOCIMIENTO} ESPECIALIZADO

Desde su inicio, la rama ejecutiva del gobierno federal se acercó a comités ciudadanos para obtener consejos -el presidente Washington creó la Comisión para la Rebelión del Whiskey (Whiskey Rebellion Commission), entre otras organizaciones, con el objetivo de suplir la débil capacidad de su gobierno para usar información en la resolución de problemas públicos. ${ }^{15}$ En 1842, el Congreso intentó limitar los gastos del gobierno en los numerosos comités consultores, pero el uso de éstos siguió en aumento a la par del creciente papel desempeñado por el gobierno federal en la economía. ${ }^{16}$ Para 1972, año en que la Ley sobre el Comité Consultivo Federal (Federal Advisory Committee Act) fundó el actual marco normativo requiriendo que la membrecía de los comités consultores fuera balanceada, su funcionamiento transparente y su duración limitada (P.L. 92-463), el gobierno federal tenía 1500 comités consultivos. Para 1978 , el número había caído a $800,{ }^{17}$ pero había vuelto a crecer para 2008 a más de $900 .{ }^{18}$ En la actualidad, el número de comités consultivos del gobierno federal excede los mil. ${ }^{19}$

Un impulso clave al uso de comités consultivos fue la dificultad de mantener conocimiento especializado dentro de la burocracia, inicialmente por la capacidad limitada de ésta y, más adelante, porque el servicio de carrera complicaba las contrataciones y los despidos, por lo que la burocracia estaba menos dispuesta a incluir personal con conocimientos especializados a corto plazo.

${ }^{15}$ Bruce L. R. Smith, The Advisors: Scientists in the Policy Process, Washington, D. C., Brookings Institution Press, 1992, p. 14.

16 Steven Croley y William Funk, "The Federal Advisory Committee Act and Good Government”, Yale Journal on Regulation, vol. 14, núm. 2, 1997, pp. 452-557.

17 Mark Petracca, "Federal Advisory Committees, Interest Groups, and the Administrative State”, Congress Eॄ The Presidency, vol. 13, núm. 1, 1986, p. 85.

18 U.S. Government Accountability Office, Federal Advisory Committees: Issues Related to Independence and Balance of Advisory Committees, GAO-08-11T, 2008, p. 1.

19 U.S. Government Services Administration, Federal Advisory Committee Act (FACA) Management Overview, http://www.gsa.gov/portal/content/104514, 5 de enero de 2015. 
La necesidad de dicho conocimiento fue particularmente urgente durante periodos de guerra. En 1863, durante la Guerra Civil, el Congreso creó la Academia Nacional de las Ciencias (National Academy of Science) bajo petición del presidente Lincoln, para aconsejar a oficinas federales en cuestiones científicas. El aumento en la demanda por conocimiento científico especializado durante la Primera Guerra Mundial llevó a la creación del Consejo Nacional de Investigación (National Research Council) en 1916 para proveer infraestructura administrativa a la Academia de las Ciencias. El Consejo Nacional de Investigación -que ahora engloba la Academia Nacional de Ciencias (National Academy of Science), la Academia Nacional de Ingeniería (National Academy of Engineering), y el Instituto de Medicina (Institute of Medicine) - hace, cada vez con mayor frecuencia, proyectos pedidos por el Congreso y por agencias administrativas. ${ }^{20}$ Estos proyectos incluyen normalmente la producción de análisis de políticas que diagnostican problemas sociales y comparan alternativas para solucionarlos.

La Segunda Guerra Mundial creó demanda no sólo por conocimiento especializado extraído de las ciencias naturales, sino también por expertise sobre cómo usar recursos escasos más eficientemente entre y adentro de organizaciones. Este conocimiento, derivado de las matemáticas, la estadística y la nueva teoría de juegos, se llamó "investigación operativa”. Motivado por el éxito analítico del "Blackett's circus" británico durante la Batalla de Inglaterra, unidades de investigación operativa se instalaron en el sector militar estadounidense. ${ }^{21}$ Trabajaban en temas disímiles: desde cuestiones de inteligencia, como la manera de usar los números de serie del equipo capturado para estimar las tasas de producción enemigas, ${ }^{22}$ hasta asuntos operativos, como el tamaño

${ }^{20}$ M. Granger Morgan y Jon M. Peha (eds.), Science and Technology Advice for Congress, Washington, D. C., Resources for the Future, 2003.

${ }^{21}$ M. Fortun y S. S. Schweber, "Scientists and the Legacy of World War II: The Case of Operations Research (OR)”, Social Studies of Science, vol. 23, núm. 4, 1993, pp. 595-642.

${ }^{22}$ Julian Champkin, “The German Tank Problem”, Significance, vol. 10, núm. 5, 2013, p. 28. 
de los envíos que maximizarían la proporción de pérdidas de submarinos frente a pérdidas de barcos mercantes. ${ }^{23}$

El sector militar de Estados Unidos mantuvo su capacidad de investigación operativa después de la guerra; además, se convirtió en el cliente principal de las organizaciones recién creadas de investigación por contrato, como la Corporación RAND (RAND Corporation). Analistas del Pentágono y de la Corporación RAND fueron recursos para implementar los Sistemas de Planeación y Presupuestación de Programas (PPBs por sus siglas en inglés) del Departamento de Defensa. Durante el proceso de planeación, los PPBs se enfocaban más en los programas que en categorías de insumos. Por ejemplo, en vez de ordenar la presupuestación por categorías de gasto, como personal o equipo agregados entre programas, los PPBs organizaban los presupuestos por objetivo de gasto, como defensa área o apoyo logístico. Los PpBs iniciaron en 1961 bajo la tutela del secretario Robert McNamara, quien había sido investigador operativo durante la guerra y presidente de la Compañía de Motores Ford. El éxito aparente de los PPBs en el Departamento de Defensa llevó al presidente Johnson a pedir en 1956 que todos los jefes de organismos gubernamentales los implementaran. No obstante, esta iniciativa tuvo cuando mucho un éxito modesto, sobre todo porque las circunstancias que permitieron beneficios en el Departamento de Defensa, incluidos capacidad analítica, información relevante y objetivos claros, no estaban presentes en la mayoría de las agencias durante esos años. ${ }^{24}$ No obstante, los esfuerzos fallidos por implementar PPBS y sus sucesores -como la Presupuestación Base-Cero (Zero-Based Budgeting) durante la administración de Carter- requirieron que los organismos incorporaran personal técnicamente capacitado, algunos de los cuales sobrevivieron estas iniciativas centralizadas y contribuyeron a desarrollar la capacidad analítica dentro de sus agencias en el largo plazo.

${ }^{23}$ Charles Hitch, "Sub-Optimization in Operations Problems", Journal of the Operations Research Society of America, vol. 1, núm. 3, 1953, pp. 87-99.

24 Aaron Wildavsky, "Rescuing Policy Analysis from PpBs", Public Administration Review, vol. 29, núm. 2, 1969, pp. 189-202. 
Varios factores incentivaron a gobiernos estatales y municipales a adoptar los ppbs. ${ }^{25}$ La Fundación Ford financió el "Proyecto de Finanzas Estatales Locales de la Universidad George Washington", que se implementó en cinco estados, cinco condados y cinco ciudades para determinar la asequibilidad y la deseabilidad de adoptar los PPBs. Este esfuerzo fue después apoyado por el Departamento de Vivienda y Desarrollo Urbano y promovido mediante incentivos en la legislación federal. Como en el caso federal, la mayoría de estas iniciativas no implementó todo el modelo PPBs y, aunque produjeron mejoras modestas en los procesos de presupuesto, no mejoraron la capacidad de los gobiernos para el análisis de políticas. ${ }^{26}$

Otro tipo de conocimiento especializado comenzó a ser demandado por el gobierno federal a finales de la década de 1960, cuando los implementadores del programa "Great Society" del presidente Johnson buscaban maneras de solucionar la pobreza. Experimentos sociales a gran escala, con una asignación estocástica de los participantes a grupos de tratamiento y control, ofrecieron la posibilidad de determinar si los programas podrían producir los resultados deseados. Desde el Experimento sobre Mantenimiento del Ingreso (Income Maintenance Experiment) de Nueva Jersey en 1968, agencias federales han contratado consultoras para realizar más de doscientos experimentos sociales. ${ }^{27}$ Estos experimentos requieren conocimiento especializado en diseño de investigación, implementación y análisis estadístico, tanto en las consultoras contratadas como en los burós que conciben, seleccionan, financian y monitorean los proyectos. Más allá de la información específica que produjeron estos experimentos, contribuyeron a la capacidad general del gobierno federal para realizar y apoyar investigación en políticas.

El New Deal expandió el papel del gobierno federal en la economía, mediante programas implementados por agencias nuevas

${ }^{25}$ Harry P. Harty, "Status of PPBs in Local and State Governments in the United States”, Policy Sciences, vol. 2, núm. 2, 1971, pp. 177-189.

${ }^{26}$ Harty, op. cit., Exhibit 1.

${ }^{27}$ David Greenberg y Mark Schroder, The Digest of Social Experiments, $3^{\mathrm{a}}$ ed., Washington, D. C., Urban Institute Press, 2004. 
y burós federales existentes, como el Departamento de Agricultura, y mediante una mayor regulación económica por parte de la Comisión de Valores (Securities and Exchange Commission, SEC) -organización que reemplazó al Buró de Corporaciones (Bureau of Corporations) -, la Comisión de Comercio Federal (Federal Trade Commission, FTC), y la Comisión de Comunicaciones Federal (Federal Communications Commission, FCC). En la década de 1970, el papel federal en salud y seguridad, la llamada "regulación social", se expandió a partir de la creación de la Administración de Seguridad Laboral y Salud (Occupation Safety and Health Administration, osha), la Comisión de Protección al Consumidor (Consumer Product Safety Commission, CPSC) y la Agencia de Protección Ambiental (Environmental Protection Agency, EPA). El sitio de Internet federal (http://www.regulations.gov) para aumentar la transparencia tiene avisos, iniciativas regulatorias, reglamentos y leyes finales, y otros materiales relacionados con la creación de normas de aproximadamente trescientas agencias federales.

El aumento en el papel del gobierno federal requirió que funcionarios afinaran los detalles de las leyes aprobadas por el Congreso y las órdenes ejecutivas expedidas por el presidente. Además, las agencias tuvieron que contratar personal con conocimiento especializado legal para diseñar y justificar regulaciones. Estos "reguladores" frecuentemente ejercían como analistas de políticas, diagnosticando problemas con reglas existentes, proponiendo alternativas y considerando las alternativas con base en los objetivos de la agencia. La Ley sobre Procedimiento Administrativo (Administrative Procedure Act) de 1946 (P.L. 79404) determinó que se publicaran en el Registro Federal las iniciativas de cambio a la regulación, lo que permite notificar a las partes interesadas sobre la iniciativa y aceptar sus comentarios antes de que la regulación sea aprobada. No obstante, buena parte del análisis de las regulaciones propuestas, sobre todo las propuestas de oficinas de gobierno y no de comisiones independientes, se hacía y todavía se hace a puerta cerrada. Esta falta de transparencia tiende a oscurecer el análisis que subyace a las propuestas públicas. La mayoría de las regulaciones son políticas públicas que requieren por lo menos un análisis mínimo, 
aunque sea sólo una comparación descriptiva frente a las políticas actuales.

Los Estados también regulan. En efecto, crearon agencias para regular profesiones, corporaciones, la industria de las aseguradoras, las utilidades públicas y algunos recursos naturales en una época en que la federación se limitaba a regular ferrocarriles mediante la Comisión de Comercio Interestatal (Interstate Commerce Commission). Aunque la regulación federal prevalece sobre la regulación estatal en áreas como transporte y finanzas, los estados emiten regulaciones, pero varían considerablemente en términos de los recursos profesionales que asignan a dichas tareas. ${ }^{28}$ Es común que asociaciones nacionales provean investigación en políticas relevante al trabajo de los reguladores estatales, como la Asociación Nacional de Comisionados de Seguros (National Association of Insurance Commissioners).

Además de aumentar el conocimiento especializado interno mediante el uso de comités auxiliares, muchas agencias federales y algunas estatales extienden sus capacidades analíticas gracias a contratos con empresas de consultoría. Estos contratos permiten a las agencias obtener recursos humanos especializados de forma temporal. En un extremo, el personal contratado puede trabajar de forma independiente para entregar un producto, como el análisis de una política o una base de datos. En el otro extremo, el personal contratado puede realizar tareas pendientes como si fuera parte de la agencia. En ambos casos, escribir y monitorear contratos eficientes requiere cierto grado de conocimiento dentro de las agencias del gobierno. Una ironía de esta privatización del análisis es que, para que sea efectiva, las agencias deben desarrollar conocimiento especializado no sólo en gestionar contratos, sino en los temas sustantivos de las políticas. ${ }^{29}$

Otra razón para contratar analistas externos es que las agencias gubernamentales deben mantener un número fijo de funcionarios,

${ }^{28}$ Paul Teske, Regulation in the States, Washington, D. C., Brookings Institution Press, 2004.

${ }^{29}$ Vining y Weimer, "Government Supply and Government Production Failure: A Framework Based on Contestability”, Journal of Public Policy, vol. 10, núm. 1, 1990, pp. 1-22. 
e incluso reducir ese número en periodos de austeridad. Durante estos periodos, es más fácil y más rápido contratar conocimiento especializado que contratar funcionarios adicionales, sobre todo al confrontarse con nuevos temas o nuevas responsabilidades de política.

El creciente uso de este tipo de contratación, referido como "gobierno de terceros, el Estado hueco, la burocracia sombra, la fuerza de trabajo pública combinada, cadenas articuladas de terceros, programas públicos y sus asociados, dirigir y no implementar", provoca preocupaciones sobre la rendición de cuentas en la administración pública de Estados Unidos. ${ }^{30}$ Esta situación significa que el contenido de muchos análisis de políticas publicados por agencias de gobierno pueden ser productos de entidades privadas. También significa que cualquier cálculo del número total de analistas de políticas en las burocracias federal y estatal subestimaría el número de personas fuera del gobierno que hacen análisis de políticas en su nombre.

En algunos casos, el Congreso ha delegado responsabilidad analítica a actores no gubernamentales. Estas delegaciones tienden a ser en áreas de política, como salud, caracterizadas por rápidos avances tecnológicos o científicos, y una complejidad que valoriza el conocimiento especializado. Por ejemplo, el Congreso dio al Comité sobre Prácticas Inmunológicas (Committee on Immunization Practices) autoridad para especificar las vacunas cubiertas por el programa de vacunación infantil, mediante el cual los Centros para el Control y la Prevención de Enfermedades (Centers for Disease Control and Prevention) hacen compras masivas de vacunas para distribuirlas a las agencias de salud estatales y locales. ${ }^{31}$

La dificultad que encaran los sistemas de carrera para mantener conocimiento especializado y de vanguardia aporta un argumento racional a favor de la delegación de tareas. Para políticas que tendrán afectados identificables, como niños que tienen efectos

${ }^{30}$ Melvin J. Dubnick y H. George Frederickson, "Accountable Agents: Federal Performance Measurement and Third-Party Government”, Journal of Public Administration Research and Theory, vol. 20, suplemento 1, 2010, p. i143.

31 Weimer, Medical Governance: Values, Expertise, and Interests in Organ Transplantation, Washington, D. C., Georgetown University Press, 2010. 
secundarios negativos por las vacunas o la asignación de órganos escasos para trasplante, los legisladores pueden guarecerse al delegar el diseño de la política a grupos de profesionales y otros actores competentes, en vez de a un buró que, a ojo de los votantes, el legislador puede sesgar a su favor. ${ }^{32}$ Un ejemplo claro de regulación de políticas por especialistas es la Red de Dotación y Transplante de Órganos (Organ Procurement and Transplantation Network, optN), que tiene la responsabilidad de definir el contenido de reglas para la procuración y atribución de órganos con consecuencias de vida o muerte. Las regulaciones deben ser emitidas por el Departamento de Salud y Servicios Humanos para tener estatus de ley. No obstante, el departamento nunca ha emitido dichas regulaciones. Por el contario, las regulaciones ganan fuerza porque el gobierno federal requiere que los centros de trasplantes sean miembros activos de la red OPTN para recibir financiamiento de Medicare, quien a su vez requiere que se ciñan a ciertas reglas. Miembros de la OPTN analizan las reglas de forma continua y las cambian de manera incremental, al contar con una base de datos longitudinal cuasi-universal de todos los reactivos de las listas de espera de trasplantes, y con su conocimiento tácito sobre trasplantes. ${ }^{33}$

Las crecientes demandas por conocimiento especializado han provocado que individuos y organizaciones fuera del gobierno participen en análisis de políticas mediante comités auxiliares, contratos y delegación de responsabilidades. Esta participación ha sido un sustituto y un complemento de la capacidad y la producción analítica dentro de los gobiernos. Al menos en el ámbito federal, esta situación ha contribuido a aumentar la cantidad y sofisticación técnica del análisis de políticas hecho de manera rutinaria por agencias de gobierno en los últimos cincuenta años.

${ }^{32}$ Weimer, "The Puzzle of Private Rulemaking: Scientific Expertise, Flexibility, and Blame Avoidance in Regulation”, Public Administration Review, vol. 66, núm. 4, 2006, pp. 569-582.

${ }^{33}$ Weimer, "Stakeholder Governance of Organ Transplantation: A Desirable Model for Inducing Evidence-Based Medicine?”, Regulation E Governance, vol. 4, núm. 3, 2010, pp. 281-302. 


\section{DEMANDA SURGIDA DE LA NEGESIDAD DE ADMINISTRAR} LA COMPLEJIDAD

El papel ampliado del gobierno en la economía no sólo creó una demanda por conocimiento especializado, sino por analistas que administren la complejidad de las políticas públicas. Tanto legisladores como miembros de la rama ejecutiva requerían información para hacer presupuestos cada vez más complejos. Nuevos programas daban una discrecionalidad considerable a administradores públicos para decidir sobre alternativas de implementación de las políticas. Como mostró en tiempos recientes la Ley para la Protección de Pacientes y Atención de Salud Asequible (Patient Protection and Affordable Act) (P.L. 111-148) de forma dramática, la complejidad en la gobernanza multinivel sigue creciendo, desde el momento en que el gobierno federal intenta inducir cambios en políticas que son competencia directa de los estados. ${ }^{34}$

\section{Apoyo analítico general para legislaturas y gobiernos}

Los esfuerzos modernos por aumentar la capacidad analítica en el gobierno federal surgieron a partir de los límites en este rubro puestos en evidencia durante la Primera Guerra Mundial. En 1921 el Congreso creó dos agencias gemelas para ofrecer apoyo neutral y competente en la mejora de la política fiscal federal: la Oficina del Presupuesto (Bureau of the Budget) (en la actualidad la Oficina de Gestión y Presupuesto, Office of Management and Budget, омв) y la Oficina General de Rendición de Cuentas (General Accounting Office, GAO) (en la actualidad Oficina Gubernamental de Rendición de Cuentas, Government Accountability Office). ${ }^{35}$

${ }^{34}$ Simon F. Haeder y David L. Weimer, "You Can't Make Me Do It! State Implementation of Insurance Exchanges under the Affordable Care Act", Public Administration Review, vol. 73, núm. 1, 2013, pp. S34-S47.

${ }^{35}$ Frederick C. Mosher, A Tale of Two Agencies: A Comparative Analysis of the General Accounting Office and the Office of Management and Budget, Baton Rouge, Louisiana State University Press, 1984. 
La Oficina del Presupuesto se enfocaba al inicio en controlar agencias presupuestales estatales, una tarea difícil cuando las agencias controlaban distritos electorales importantes en el Congreso, pero después adquirió el papel de "árbitro" y "látigo". El primer papel implicaba resolver disputas jurisdiccionales entre agencias; el segundo implicaba cortar fondos de valor cuestionable añadidos al presupuesto para agradar a ciertos grupos de votantes; miembros del Congreso obtenían reconocimiento por las inserciones y podían culpar a la Oficina del Presupuesto por removerlas. ${ }^{36}$ En 1970 la Oficina del Presupuesto se convirtió en la Oficina de Gestión y Presupuesto. Un aumento en el número de políticos designados para su administración mermó la reputación de neutralidad de esta Oficina, pero sirvió para que desempeñara un mayor papel analítico en el desarrollo de políticas durante las administraciones siguientes. En la actualidad emplea aproximadamente a 470 personas.

La Oficina General de Rendición de Cuentas (GAO) fungió en sus inicios como una auditoría de las transacciones financieras de las agencias. El volumen inmenso de dichas transacciones durante la Segunda Guerra Mundial la llevó a enfocarse en procedimientos y prácticas de las agencias, con que proveyó información al Congreso sobre procedimientos de las agencias, que de otra forma serían opacos, mediante reportes sobre análisis de políticas accesibles al público y que incluían recomendaciones. El Congreso reforzó la función de análisis de políticas de la GAO mediante la Ley de Reorganización Legislativa (Legislative Reorganization Act) de 1970 (P.L. 91-510), el cual ordena a la GAO realizar evaluaciones de programas y análisis costo-beneficio. La GAO emplea casi tres mil personas en la actualidad, muchas de las cuales hacen análisis de políticas. En un año típico, esta Oficina emite casi 1500 recomendaciones específicas para mejorar las operaciones de gobierno; reporta, además, que $79 \%$ de las recomendaciones que hizo durante el año fiscal 2009 fueron implementadas para el final del

${ }^{36}$ Patrick J. Wolf, "Neutral and Responsive Competence: The Bureau of the Budget, 1939-1948 Revisited”, Administration and Society, vol. 31, núm. 1, 1999, pp. 142-167. 
año fiscal 2013. ${ }^{37}$ Aunque la GAO ha influido en diversas áreas de políticas, las afirmaciones sobre su propia eficacia pueden ser demasiado optimistas, porque sus recomendaciones seguido provienen de las agencias cuyos programas están siendo evaluados.

La Ley de Reorganización Legislativa también dio mayor independencia, y responsabilidad de aportar análisis de políticas para apoyar a comités legislativos, al Servicio de Investigación Legislativa de la Biblioteca del Congreso (Legislative Research Service of the Library of Congress), y le cambió el nombre a Servicio de Investigación del Congreso (Congressional Research Service, CRs). ${ }^{38}$ El CRS emplea alrededor de seiscientas personas y realiza análisis para comités y miembros individuales del Congreso. El perfil discreto pero de alto nivel de su servicio a miembros del Congreso ha contribuido a su estabilidad, lo que le permite fungir como depositario de la "memoria institucional". En el año fiscal 2012 reportó haber respondido a más de setenta mil solicitudes de análisis, información e investigación. ${ }^{39} \mathrm{Al}$ contrario de la GAO, el CRS generalmente no emite recomendaciones y sólo selectivamente hace públicos sus reportes.

Las disputas con la administración Nixon por la retención de fondos consignados por el Congreso llevó a éste a fortalecer su capacidad de analizar presupuestos. Creó la Oficina Presupuestal del Congreso (Congressional Budget Office, сво) mediante la Ley de Presupuesto Legislativo y Control de Retención de Fondos (Congressional Budget and Impoundment Control Act) de 1974 (P.L. 94344). La сво se organiza en ocho divisiones, siendo la mayor la División de Análisis Presupuestario, que analiza los presupuestos del presidente y determina las implicaciones fiscales de propuestas legislativas, entre otras funciones. De los 235 empleados aproximados, más de la mitad están en una plaza denominada "analista",

${ }^{37}$ U.S. Government Accountability Office, Summary of GAO's Performance and Accountability Report: Fiscal Year 2014, GAO-14-3SP, 2014.

${ }^{38}$ Walter Kravitz, "The Advent of the Modern Congress: The Legislative Reorganization Act of 1970”, Legislative Studies Quarterly, vol. 15, núm. 3, 1990, pp. 375-399.

${ }^{39}$ U.S. Congressional Research Service, Annual Report of the Congressional Research Service of the Library of Congress for Fiscal Year 2012, Washington, D. C., Congressional Research Service, 2013. 
y la mayoría se enfoca en el impacto fiscal y presupuestario de las políticas.

Una cuarta organización, la Oficina de Valoración Tecnológica (Office of Technology Assessment, оTA), aportaba análisis al Congreso sobre asuntos con importantes componentes tecnológicos o científicos. Creada en 1972 (P.L. 92-484), se eliminó en 1995 a la par de las reducciones del personal de todas las agencias de apoyo legislativo, después de que los republicanos obtuvieron la mayoría en la Cámara de Diputados y desearon reflejar su compromiso con la reducción del déficit. Un factor de la caída de la отA fue que el Congreso podía obtener fácilmente análisis sustitutos de universidades, think tanks y asociaciones profesionales. Otro factor del cierre de la отA puede haber sido que sus análisis, hechos por especialistas sin conexiones con los operadores de las políticas, a veces desafiaban las líneas preferidas de gremios poderosos, de manera que perdían el apoyo de representantes que simpatizaban con esos intereses y que no dependían de la отA para el tipo de apoyo cotidiano ofrecido por las otras tres agencias de apoyo legislativo. ${ }^{40}$

Parece que la GAO, la CRS y la CBO han tenido éxito y se han forjado una reputación de proveer análisis que personifican la neutralidad competente, que Kaufman define como "la habilidad para hacer el trabajo del gobierno adecuadamente, y hacerlo siguiendo estándares explícitos y objetivos en vez de seguir obligaciones y lealtades personales o partidistas". ${ }^{41}$

$\mathrm{Al}$ contrario de las agencias administrativas que mantienen la responsabilidad de promover la agenda de políticas del presidente, estas agencias legislativas están diseñadas para apoyar a los partidos mayoritarios y minoritarios en cada cámara. Como el control de las cámaras puede variar incluso cada dos años, la neutralidad, y la percepción de neutralidad, reduce las probabilidades de que cambios en el control partidista amenacen la existencia de las agencias. La percepción de neutralidad también se consigue al tener

${ }^{40}$ Bruce Bimber, The Politics of Expertise in Congress: The Rise and Fall of the Office of Technology Assessment, Albany, State University of New York Press, 1996.

${ }^{41}$ Herbert Kaufman, "Emerging Conflicts in the Doctrines of Public Administration", American Political Science Review, vol. 50, núm. 4, 1956, p. 1060. 
personal profesional estable, y por la designación de directivos no partidistas con cargos de larga duración..$^{42}$ Por ejemplo, el director de la сво es designado por cuatro años y el Contralor General que dirige la GAO es designado por un periodo de quince años. Aunque el director del CRS no tiene un plazo fijo, el Bibliotecario del Congreso, y no los líderes de las cámaras, lo designa en consulta con el Comité de la Biblioteca. ${ }^{43}$

Los estados han creado agencias para apoyar el trabajo legislativo análogas a las que operan a nivel federal. En algunos casos los estados incluso abrieron brecha en este sentido. La primera organización profesional y no partidista que proveyó apoyo de redacción e investigación a una legislatura estatal fue el Buró de Referencia Legislativa de Wisconsin (Wisconsin Legislative Reference Bureau), creado en 1901. Pocos estados le siguieron durante los siguientes cuarenta años, pero en 1941 California creó la Oficina de Análisis Legislativo (Legislative Analyst's Office), con capacidad analítica sustantiva, que sirvió de modelo para la creación de oficinas similares en otros estados e incluso para la Сво. Además de proveer servicios similares a la Сво у al CRS, también mantiene memoria institucional relevante sobre los debates de políticas, una función que ha crecido en importancia desde la adopción de límites a los tiempos de los legisladores. ${ }^{44}$ Basado en información del Consejo de Gobernadores de Estados (Council of State Governments) y de la Conferencia Nacional de Legislaturas Estatales (National Conference of State Legislature), Hird identificó 105 agencias no partidistas que dan apoyo analítico y de investigación a legislaturas estatales. ${ }^{45}$ De ochenta agencias que respondieron preguntas sobre sus

42 Weimer, "Institutionalizing Neutrally Competent Policy Analysis: Resources for Promoting Objectivity and Balance in Consolidating Democracies”, Policy Studies Journal, vol. 33, núm. 2, 2005, pp. 131-146.

${ }^{43}$ Ida A. Brudnick, "Legislative Branch Agency Appointments: History, Processes, and Recent Proposals", CRS Report for Congress, Washington, D. C., Congressional Research Service, 2013.

${ }^{44}$ Beryl Radin, Beyond Machiavelli: Policy Analysis Reaches Midlife, $2^{\mathrm{a}}$ ed., Washington, D. C., Georgetown University Press, 2013.

${ }^{45}$ John A. Hird, Policy Analysis in the States: Power, Knowledge, and Politics, Washington, D. C., Georgetown University Press, 2005. 
actividades, $81 \%$ reportó que el análisis de políticas era una de las principales.

Una organización particularmente influyente que apoya la formulación de políticas de una legislatura estatal es la agencia no partidista Instituto de Políticas Públicas del Estado de Washington (Washington State Institute for Public Policy, wsipP). Además de sus esfuerzos por identificar y valorar distintas alternativas a la expansión carcelaria, el wsipp ha realizado numerosos meta-análisis de evaluaciones de distintos tipos de programas. Este instituto usa medidas de estimación de impacto para hacer análisis costo-beneficio sofisticados e influyentes sobre portafolios de programas para la legislatura del estado de Washington. ${ }^{46}$ En los últimos cuatro años, dieciséis estados han adoptado la herramienta del wispP mediante la Iniciativa "Resultados Primero" (Results First Initiative), ${ }^{47}$ un esfuerzo conjunto de la Fundación MacArthur y los Pew Charitable Trusts. La iniciativa ayuda a los estados a organizar actores interesados en grupos para usar los análisis costo-beneficio, y ofrece asistencia técnica para implementar la herramienta wispP con datos específicos a nivel estatal.

Algunas ciudades grandes también han creado organizaciones no partidistas que elaboran análisis en un rango amplio de temas sustantivos. El ejemplo más prominente es la Oficina Independiente de Presupuesto (Independent Budget Office) de la ciudad de Nueva York. Creada en 1989 durante una revisión exhaustiva del estatuto orgánico de la ciudad, produce resúmenes temáticos y otros análisis de políticas, además de cumplir con sus responsabilidades de proyección fiscal y proveer evaluaciones sobre los presupuestos preliminares y finales.

Aunque una evaluación de la prevalencia de dichas agencias a nivel de los gobiernos locales no está disponible, parece que se han generalizado actividades para la medición del desempeño. ${ }^{48}$

46 Aidan R. Vining y David L. Weimer, "Assessing the Costs and Benefits of Social Policies", en D. Weimer y A. Vining (eds.), Investing in the Disadvantaged: Assessing the Benefits and Costs of Social Programs, Washington, D. C., Georgetown University Press, 2009, pp. 1-16.

${ }^{47}$ http:/ / www.pewtrusts.org/pew-macarthur-results-first-iniciative

48 Julia Melkers y Katherine Willoughby, "Models of Performance-Measure- 
Apoyo analítico dentro de las agencias

Todas las agencias de gobierno federales tienen áreas que elaboran análisis de políticas en apoyo de los objetivos de la organización. Antes de la Segunda Guerra Mundial, los directivos de las agencias recurrían a sus departamentos legales para el análisis de políticas correspondiente a toda la agencia; después comenzaron a crear oficinas especializadas en aportar ese análisis. Por ejemplo, durante la década de 1930, el Ministerio del Interior recurría a la Oficina del Procurador General para el análisis de políticas; fue hasta 1947 que se creó un área para el análisis de políticas, que más adelante se convirtió en la Oficina de Análisis de Políticas en $1974 .{ }^{49}$

El papel de coordinación a nivel departamental es particularmente importante. Por ejemplo, consideremos la Oficina del Subsecretario de Planeación y Evaluación en el enorme y complejo Departamento de Salud y Servicios Humanos. Los analistas desempeñan un papel en el desarrollo de las políticas, incluyendo la definición de alternativas y la evaluación de sus consecuencias. Además de tener papeles en monitoreo e investigación y otras responsabilidades más evidentes, como resolver "bomberazos" -la respuesta inmediata a requerimientos de información sobre asuntos urgentes-, la guía para nuevos empleados de esta oficina identifica el papel de "oficial encargado", que implica coordinar políticas asociadas a ciertas áreas programáticas. ${ }^{50}$ Un miembro del personal con una larga trayectoria opinó: "La coordinación con otros analistas de la oficina del presupuesto, la oficina legislativa, y de otras oficinas es crítica. Los analistas no aprecian ser una voz disidente y solitaria en un tema de política". ${ }^{51}$ En muchas circunstancias, los

ment Use in Local Governments: Understanding Budgeting, Communication, and Lasting Effects", Public Administration Review, vol. 65, núm. 2, 2005, pp. 180-190.

${ }^{49}$ Robert H. Nelson, "The Office of Policy Analysis in the Department of the Interior”, Journal of Policy Analysis and Management, vol. 8, núm. 3, 1989, pp. 395-410.

${ }^{50}$ Assistant Secretary for Policy and Evaluation, All about ASPE: A Guide for ASPE Staff, n.d.

51 George D. Greenberg, "Policy Advice at the Department of Health and Human Services: Then and Now", Journal of Policy Analysis and Management, vol. 22, núm. 2, 2003, pp. 304-307. 
funcionarios públicos deben desempeñar el papel de analistas de políticas, al elegir entre alternativas de implementación que permitan cumplir los objetivos de sus agencias; en las oficinas analíticas de los grandes Departamentos, parece que los analistas deben desempeñar el papel de administradores para ser eficientes, al coordinarse con otros analistas y personal de los programas.

En las principales oficinas de los gobiernos estatales también hay personal encargado de hacer análisis de políticas que cumple funciones similares a los analistas en el ámbito federal. El uso extensivo de organizaciones con y sin fines de lucro para proveer servicios sociales implica que la información relevante para las políticas, e incluso la capacidad analítica, tiende a distribuirse dentro de redes de políticas, conformadas por diversas organizaciones con objetivos comunes. ${ }^{52}$ Podemos plantear la hipótesis de que el papel de "oficial encargado" para los analistas en las agencias estatales ha crecido en importancia a la par del aumento en la provisión de servicios sociales por otras organizaciones. Debemos también reconocer que algunas organizaciones dentro de las redes de políticas pueden ser fuentes primarias, o incluso colaboradores, del análisis de políticas.

Las redes de políticas también pueden moldear la etapa de difusión de las políticas en múltiples jurisdicciones, al ofrecer análisis que defensores pueden usar durante la etapa de discusión de alternativas, y por administradores durante la etapa de implementación. Por ejemplo, información producida por organizaciones locales, estatales y nacionales ha desempeñado un papel vital en difundir el uso de tribunales especializados en drogas, los cuales buscan romper el vínculo entre abuso de sustancias y comportamiento criminal mediante un mayor uso de tratamientos en vez de castigos. La Asociación Nacional de Profesionistas de los Tribunales de Drogas (National Association of Drug Court Professionals), una organización privada, promovió estos tribunales mediante una campaña de información intensiva, lo

${ }^{52}$ H. Brinton Milward y Keith G. Provan, "Governing the Hollow State”, Journal of Public Administration Research and Theory, vol. 10, núm. 2, 2000, pp. 359-380. 
que llevó a su adopción en más de 2000 jurisdicciones desde 1989.53

\section{DEMANDA POR LA VOLUNTAD DE LOS POLÍTICOS DE DISCIPLINAR SUS ACCIONES (¿Y LAS DE SUS COLEGAS?)}

La imposición de reglas de procedimiento en procesos de toma de decisión por políticos ha sido vista normativamente como una forma de asegurar equidad ${ }^{54}$ y positivamente como una forma de controlar los burós mediante la obligación de generar información y la emancipación de grupos de votantes específicos. ${ }^{55}$ En el caso de requerimientos para la producción rutinaria de análisis de políticas, también podemos considerar que los políticos contrarrestan incentivos futuros para apoyar políticas defendidas por sus votantes pero que no necesariamente corresponden al interés público. Pueden estar dispuestos a amarrarse las manos y, quizás más importante para ellos, las manos de sus futuros colegas por la incertidumbre sobre qué políticas serán consideradas en el futuro. A pesar de que el costo político de renunciar a un beneficio para sus votantes puede ser alto, el costo político percibido de adoptar una regulación que haga más difícil esa deferencia hacia sus votantes en el futuro puede ser bajo, por el "velo de la ignorancia" sobre qué demandas futuras se materializarán. ${ }^{56}$ En otras palabras, los políticos que favorecen la implementación de análisis en términos generales pueden oponerse a análisis de casos específicos cuando anticipan que éstos no favorecerán sus políticas de preferencia. No obstante, pueden aceptar el riesgo de que ciertos análisis en el

${ }^{53}$ Kathleen Hale, How Information Matters: Networks and Public Policy Innovation, Washington, D. C., Georgetown University Press, 2011.

${ }^{54}$ Richard B. Stewart, "The Reformation of Administrative Law", Harvard Law Review, vol. 88, núm. 8, 1975, pp. 1669-1813.

55 Mathew D. McCubbins, Roger G. Noll y Barry R. Weingast, "Administrative Procedures as Instruments of Political Control”, Journal of Law, Economics and Organization, vol. 3, núm. 2, 1987, pp. 243-277.

${ }^{56}$ Weimer, "Claiming Races, Broiler Contracts, Heresthetics, and Habits: Ten Concepts for Policy Design”, Policy Sciences, vol. 25, núm. 2, 1992, pp. 135-159. 
futuro no los favorecerán, porque en ocasiones les ayudarán a prevenir políticas a las cuales se oponen.

El Congreso y el presidente han aprobado reglas de procedimiento que obligan a realizar análisis en ciertas circunstancias. El proceso de declaración de impacto ambiental es un ejemplo prominente de una regla impuesta por el Congreso a favor de los análisis. Corresponde a la perspectiva convencional sobre los requisitos de procedimiento como mecanismos para empoderar a grupos de electores, pero también puede ser analizado como una situación en la que el Congreso impone los análisis requeridos, y que deberán ser explícitamente revocados en legislaciones futuras. Un ejemplo más mundano es el requisito de estimaciones de costo para leyes en trámite.

Bajo la Ley Nacional de Políticas de Medio Ambiente (National Environmental Policy Act) adoptada en 1970 (P.L. 91-190), las agencias federales deben preparar declaraciones de impacto ambiental antes de implementar cualquier acción que podría afectar significativamente el ambiente. Aunque las declaraciones de impacto ambiental necesitan recursos analíticos sustantivos, se considera que tienen varias deficiencias que merman su valor como análisis de políticas, incluida la ausencia de una comparación de alternativas y considerar sólo un rango limitado de impactos. ${ }^{57}$ No obstante, la preparación de declaraciones de impacto ambiental involucra a analistas en agencias y empresas de consultoría, además de los grupos de interés que deciden enfrentárseles durante los procedimientos regulatorios o ante un tribunal.

El Congreso buscó amarrarse las manos respecto a los proyectos de gestión del agua, que históricamente eran uno de los ingredientes más comunes de legislaciones clientelistas. Desde el inicio del siglo pasado, el Congreso demandó predicciones de costos y beneficios para iniciativas de proyectos, y para mediados del siglo requirió al Cuerpo de Ingenieros del Ejército (Army Corps of Engineers) que

${ }^{57}$ Robin Gregory, Ralph Keeney y Detlof von Winterfeldt, "Adapting the Environmental Impact Statement Process to Inform Decisionmakers”, Journal of Policy Analysis and Management, vol. 11, núm. 1, 1992, pp. 58-75. 
realizara análisis costo-beneficio. ${ }^{58}$ La imposición de tener que demostrar resultados positivos hizo más fácil detectar y desechar los peores proyectos. El proceso se volvió suficientemente importante como para que la Oficina del Presupuesto (Bureau of the Budget) emitiera lineamientos sobre el uso de los análisis costobeneficio en proyectos acuíferos y agrarios relacionados a la gestión del agua. ${ }^{59}$

El análisis costo-beneficio se ha convertido en un aspecto vital de la regulación de las agencias. Evolucionó a partir de una serie de decretos del ejecutivo cuya finalidad era obligar a las agencias a considerar más sistemáticamente las consecuencias de sus iniciativas regulatorias. ${ }^{60} \mathrm{El}$ presidente Nixon creó un sistema llamado "Quality of Life" para monitorear regulaciones en temas de medio ambiente, seguridad del consumidor y del trabajador, y salud pública, a las cuales supervisaba la Oficina de Gestión y Presupuesto. ${ }^{61}$ De la misma forma, el presidente Ford decretó que las agencias consideraran el impacto de las regulaciones en la inflación (Executive Order 11821), y el presidente Carter decretó que las agencias condujeran análisis de costo-eficiencia de regulaciones propuestas (Executive Order 12044).

El uso del análisis costo-beneficio para las regulaciones más importantes de las agencias federales empezó con el presidente Reagan, en 1981 (Executive Order 12291). Una regulación que involucre más de cien millones de dólares de gastos anuales requiere de la evaluación de sus costos y beneficios mediante un Análisis de Impacto Regulatorio (Regulatory Impact Analysis, RIA) y está sujeto a revisión por la Oficina de Gestión y Presupuesto. Los RIA, que por lo general tienen un centenar de páginas, se resumen en las regulaciones publicadas en el Registro Federal.

${ }^{58}$ Richard J. Hammond, "Convention and Limitation in Benefit-Cost Analysis”, Natural Resources Journal, vol. 6, núm. 2, 1966, pp. 195-222.

${ }^{59}$ Bureau of the Budget, Circular No. A-47, 31 de diciembre de 1952.

${ }^{60}$ Curtis, W. Copeland, Cost-Benefit and Other Analysis Requirements in the Rulemaking Process, Washington, D. C., Congressional Research Service, 30 de agosto de 2011.

${ }^{61}$ George P. Shultz, Memorandum for the Heads of Departments and Agencies, Washington, D. C., Office of Management and Budget, 5 de octubre de 1971. 
Los presidentes después de Reagan han mantenido la obligación de producir análisis costo-beneficio y han aumentado el rango y los requerimientos analíticos de los RIA. El presidente Clinton expandió las reglas sujetas a análisis más allá del tope de gastos anuales de cien millones de dólares, al crear la categoría de "regulaciones económicamente significativas" -regulaciones que potencialmente afectan de forma material un sector de la economía, productividad, competencia, empleo, medio ambiente, salud pública o seguridad, o gobiernos estatales o locales (Executive Order 12866). El Consejo de Asesores Económicos del presidente G. W. Bush formalizó los lineamientos de la Oficina de Gestión y Presupuesto (омв por sus siglas en inglés) para realizar análisis costo-beneficio, incluyendo el requisito de que se tome en cuenta un número razonable de alternativas a la regulación propuesta. ${ }^{62} \mathrm{El}$ presidente Obama expandió el alcance de los análisis costo-beneficio al obligar a las agencias a realizar evaluaciones ex post para determinar si la continuación de ciertas regulaciones está justificada económicamente (Executive Order 13563), y a someter sus planes a revisión de la омв (Executive Order 13610).

Como los decretos conciernen sólo a las agencias administrativas, las comisiones reguladoras independientes (las agencias que adoptan regulaciones mediante un voto mayoritario de comisionados designados por mandatos fijos), como la FCC y la SEC, no requieren realizar RIA. El Congreso intentó expandir el requerimiento de los RIA a las agencias reguladoras independientes mediante una previsión en la Ley de Mejora "Contract with America" (Contract with America Advancement Act) (P.L. 104-121) al obligarlas a someter resúmenes de los análisis ante el Contralor General. Parece ser, sin embargo, que estos resúmenes son más de forma que de fondo, y en su mayoría contienen poca información sobre cómo se realizan los análisis costo-beneficio. ${ }^{63} \mathrm{El}$ presidente Obama instó a las comisiones

62 Office of Management and Budget, "Regulatory Analysis", OMB Circular A-4, Washington, D. C., Office of Management and Budget, 17 de septiembre de 2003.

${ }^{63}$ Edward Sherwin, "The Cost-Benefit Analysis of Financial Regulation: Lessons from the SEC's Stalled Mutual Fund Reform Effort", Stanford Journal of Law, Business, and Finance, 12, 2006, pp. 1-60. 
reguladoras independientes a elaborar los análisis que son obligatorios para las otras agencias (Executive Order 13579).

El Congreso impuso otros requerimientos analíticos a la elaboración de regulaciones. La Ley de Flexibilidad Regulatoria (Regulatory Flexibility Act) (P.L. 96-254) busca forzar a las agencias a anticipar los efectos de las regulaciones en entidades pequeñas, como negocios con pocos empleados y gobiernos locales con escasa población. La Ley de Reducción de Papeleo (Paperwork Reduction Act) (P.L. 104-13) obliga a las agencias a justificar la obtención de datos de la población. La Ley de Reforma de Mandatos No Financiados (Unfunded Mandates Reform Act) (P.L. 104-4) requiere puntualmente análisis de regulaciones que involucran mandatos costosos al sector privado, gobiernos estatales o locales, o tribus. No obstante, como sus previsiones se superponen con los requerimientos del RIA, no se necesitan análisis adicionales para defender las iniciativas de regulación bajo esta Ley.

La conformidad frente a los requisitos de procedimiento marca la pauta para el desarrollo de capacidades analíticas dentro del gobierno. En la medida en que esos análisis se vuelven influyentes, por proveer información durante la discusión de alternativas de política o por justificar la elección final, varios actores fuera del gobierno tienen el incentivo de invertir en la capacidad de al menos criticar dichos análisis.

ReSPUESTA DE LA OFERTA ANTE LAS DEMANDAS POR ANÁLISIS DE POLÍTICAS

Antes de la década de 1970, los que iniciaban una carrera como analistas de política lo hacían con un entrenamiento desde distintos campos de estudio, incluidos la administración pública, derecho, programas marcadamente orientados a la profesionalización -como planeación urbana y salud pública-, investigación operativa, estadística, negocios y las disciplinas de las ciencias sociales. En particular, dentro de la administración pública y las disciplinas "profesionalizantes" se crearon especialidades en análisis de políticas para enfocar el entrenamiento en la resolución de problemas. 
A partir de finales de la década de 1960 se establecieron programas de posgrado en universidades prominentes de Estados Unidos diseñados para dar una preparación específica en análisis de políticas. Estos programas, que se crearon como alternativas a los programas tradicionales en administración pública enfocados en "los aspectos institucionales y operativos del gobierno", ${ }^{64}$ hacían énfasis en los enfoques interdisciplinarios de diseño e implementación de políticas. En 1973, donaciones de la Fundación Ford y de la corporación RAND otorgadas a siete programas en políticas públicas de importantes universidades dieron un impulso decisivo a la creación de programas independientes en este tema. La Fundación Ford decidió "eludir las escuelas tradicionales de administración pública y promover nuevos programas en políticas públicas -ya encaminados", lo que mostraba la convicción de que "una combinación de ciencias sociales puede ser diseñada para educar a la gente a analizar y gestionar problemas complejos". ${ }^{65}$

La diferencia entre programas en administración pública y programas en análisis de políticas disminuyó significativamente con el paso de los años. El entrenamiento en análisis de políticas aumentó en las escuelas de administración pública, hasta que en 1974 la Asociación Nacional de Escuelas en Asuntos Públicos y Administración Pública (National Association of Schools of Public Affairs and Administration) la reconoció como un área de estudio prominente de la disciplina. Al mismo tiempo, dos factores impulsaron a las nuevas escuelas en análisis de políticas a asemejarse a programas de administración pública. Primero, la planta docente de estas escuelas reconoció la importancia de la implementación para determinar si las políticas producen los resultados esperados. Los procesos organizacionales y liderazgo, temas tradicionales en el campo de la administración pública, son cruciales para la implementación; los administradores deben llenar los detalles dejados en blanco durante la etapa de diseño de las políticas al momento

64 Ernest A. Engelbert, "University Education for Public Policy Analysis", $P u$ blic Administration Review, vol. 37, núm. 3, 1977, pp. 228-229.

${ }^{65}$ Ford Foundation, Ford Foundation Program in Public Policy and Social Organization, Nueva York, Ford Foundation, 1976, p. 5. 
de ponerlas en práctica, determinar opciones para el cambio y sus consecuencias, y tomar la iniciativa de cambiar las políticas cuando no parecen producir los resultados deseados. Segundo, los incentivos del mercado universitario que percibían muchas escuelas nuevas los llevaron a introducir especialidades en gestión pública. Con esto buscaron expandir el mercado de sus estudiantes más allá del pequeño número de universitarios interesados en carreras de análisis de políticas y preparados cuantitativamente para desarrollar las habilidades técnicas demandadas por los puestos de trabajo en este campo.

Las nuevas escuelas en políticas públicas, a la par de varias organizaciones de investigación, fundaron la Asociación de Análisis y Gestión de Políticas Públicas (Association for Public Policy Analysis and Management) en 1979 para promover la práctica y enseñanza del análisis de políticas y de la gestión pública. Hoy en día, entre sus miembros institucionales hay más de setenta programas de entrenamiento basados en universidades estadounidenses. La Asociación patrocina el Journal of Policy Analysis and Management y organiza conferencias anuales de investigación que atraen tanto a académicos como a practicantes. Otras organizaciones, incluyendo la Asociación para el Análisis de Riesgo (Society for Risk Analysis) y la Asociación para el Análisis Costo-Beneficio (Society for BenefitCost Analyses), también buscan atraer a los que enseñan e implementan esos tipos específicos de análisis de políticas.

\section{CONCLUSión}

Las cuatro demandas por análisis de política no se reducirán. En efecto, podemos asegurar que las demandas provenientes de la necesidad de conocimiento especializado y de la gestión de la complejidad del sector público continuarán aumentando. Además, la capacidad analítica que surge de estas demandas se ha enraizado en las organizaciones dentro y fuera del gobierno. En consecuencia, es improbable que la prevalencia del análisis de políticas en Estados Unidos disminuya, y puede fácilmente seguir aumentando. Pero, en un ambiente de un discurso público burdo y marcada polarización 
partidista, uno puede preguntarse si el análisis de políticas contribuye efectivamente a políticas públicas que mejoran la sociedad. Los análisis de políticas abundan, ¿pero con qué resultados?

Los gobiernos rutinariamente se enfrentan a decisiones sobre cómo usar recursos escasos. En lo común, estas decisiones no implican gran controversia política. Podemos estar en desacuerdo sobre si el rechazo debiera ser recogido por empleados públicos que trabajan para el sector público o por empleados privados que trabajan para un contratista privado. Podemos incluso estar en desacuerdo sobre el peso que se debe dar a la pertinencia, fiabilidad y costo-eficiencia de los servicios. No obstante, es probable que valoremos análisis que nos ayuden a entender los compromisos de cada alternativa de política. En algunos casos, a pesar de nuestras diferencias partidistas o ideológicas, podríamos incluso querer que decisiones importantes sean tomadas por analistas neutros y competentes; mi hipótesis es que la mayoría de nosotros queremos que decisiones sobre cómo minimizar el daño causado por enfermedades infecciosas refleje conocimiento epidemiológico.

Aunque parece haber mayor voluntad en el ambiente polarizado de la actualidad para cuestionar las estadísticas oficiales y las predicciones que otrora se aceptaban sin discusión, hay todavía una predisposición a tomar algunos análisis como supuestos comunes en presupuestación y otras áreas de políticas. Las proyecciones y estimaciones de costo de las agencias de apoyo legislativo, como la сво, pueden ser aceptadas por la credibilidad o la consistencia de su fuente, o simplemente porque sin algunos supuestos comunes la formulación de políticas sería demasiado caótica.

El proceso federal de creación de regulaciones es la base del contenido sustantivo de las políticas públicas en Estados Unidos. Los análisis que deben hacerse durante dicho proceso, desgraciadamente, no determinan la decisión final sobre estas regulaciones. No obstante, los análisis mejoran el valor social de las reglas adoptadas, al poner énfasis en la consideración de alternativas y al ayudar a desechar las que tienen beneficios negativos netos. Las agencias han desarrollado capacidades para cumplir con los requisitos de análisis; esto tiene externalidades positivas hacia otras funciones de las agencias, como el diagnóstico pertinente de problemas a atender. 
Muchos políticos han adoptado la retórica sobre el valor de la formulación de políticas basada en evidencia. Por supuesto, el buen análisis de políticas está basado en evidencia, cuando existe información relevante o cuando ésta puede ser recolectada dentro de los límites en los que se produce el análisis. Afortunadamente, la gran apuesta por la investigación en políticas en Estados Unidos genera una cantidad considerable de evidencia relevante para las políticas, por efecto de evaluaciones experimentales y cuasi-experimentales de programas, experimentos naturales y estudios creativos basados en la observación. La revolución digital ya ha reducido los costos de encontrar evidencia, y el "big data" puede proveer nuevas formas de crearla. Cuando existe información relevante, la retórica de la formulación de políticas basada en evidencia puede fungir de puente entre diferencias ideológicas. En la actualidad, esto puede estar sucediendo en la justicia criminal. Tanto conservadores como liberales comparten el objetivo de reducir el crimen. No obstante, los conservadores tienden a favorecer métodos de castigo como el aislamiento, la disuasión y la retribución, mientras que los liberales han tendido a favorecer la rehabilitación. Hoy en día, los crecientes costos del sistema penitenciario han hecho que conservadores de varios estados se abran a considerar alternativas a largos periodos de cárcel; las tasas de reincidencia han hecho que liberales se preocupen por cómo se utilizan los fondos cada vez más escasos de los programas. Identificar intervenciones basadas en evidencia que reduzcan los costos de encarcelamiento y la reincidencia pueden ser políticas que ganen apoyo a lo largo del espectro ideológico.

En conclusión, es probable que la lógica de las demandas históricas por análisis de políticas en Estados Unidos continúe. Lograr que estos análisis sean más útiles debiera ser una preocupación constante de los académicos especialistas en asuntos públicos. 


\section{BibliogRAFíA}

Assistant Secretary for Policy and Evaluation, All about ASPE: A Guide for ASPE Staff, n.d.

Bertelli, Anthony. M. y Laurence E. Lynn, Jr., Madison's Managers: Public Administration and the Constitution, Baltimore, The Johns Hopkins University Press, 2006.

Bimber, Bruce, The Politics of Expertise in Congress: The Rise and Fall of the Office of Technology Assessment, Albany, State University of New York Press, 1996.

Brudnick, Ida A., "Legislative Branch Agency Appointments: History, Processes, and Recent Proposals", CRS Report for Congress, Washington, D. C., Congressional Research Service, 2013.

Bureau of the Budget, Circular No. A-47, 31 de diciembre de 1952.

Champkin, Julian, "The German Tank Problem”, Significance, vol. 10, núm. 5, 2013, p. 28.

Copeland, Curtis, W., Cost-Benefit and Other Analysis Requirements in the Rulemaking Process, Washington, D. C., Congressional Research Service, 30 de agosto de 2011.

Croley, Steven y William Funk, "The Federal Advisory Committee Act and Good Government”, Yale Journal on Regulation, vol. 14, núm. 2, 1997, pp. $452-557$.

Dubnick, Melvin J. y H. George Frederickson, “Accountable Agents: Federal Performance Measurement and Third-Party Government", Journal of Public Administration Research and Theory, vol. 20, suplemento 1, 2010, pp. i143-i159.

Engelbert, Ernest A., "University Education for Public Policy Analysis", Public Administration Review, vol. 37, núm. 3, 1977, pp. 220-236.

Ford Foundation, Ford Foundation Program in Public Policy and Social Organization, Nueva York, Ford Foundation, 1976.

Fortun, M. y S. S. Schweber, "Scientists and the Legacy of World War II: The Case of Operations Research (OR)", Social Studies of Science, vol. 23, núm. 4, 1993, pp. 595-642.

Gill, Norman N., Municipal Research Bureaus: A Study of the Nation's CitizenSupported Agencies, Washington, D. C., American Council on Public Affairs, 1944. 
Greenberg, David y Mark Schroder, The Digest of Social Experiments, $3^{\mathrm{a}}$ ed., Washington, D. C., Urban Institute Press, 2004.

Greenberg, George D., "Policy Advice at the Department of Health and Human Services: Then and Now", Journal of Policy Analysis and Management, vol. 22, núm. 2, 2003, pp. 304-307.

Gregory, Robin, Ralph Keeney y Detlof von Winterfeldt, "Adapting the Environmental Impact Statement Process to Inform Decisionmakers", Journal of Policy Analysis and Management, vol. 11, núm. 1, 1992, pp. 58-75. Haeder, Simon F. y David L. Weimer, "You Can't Make Me Do It! State Implementation of Insurance Exchanges under the Affordable Care Act", Public Administration Review, vol. 73, núm. 1, 2013, pp. S34-S47.

Hale, Kathleen, How Information Matters: Networks and Public Policy Innovation, Washington, D. C., Georgetown University Press, 2011.

Hammond, Richard J., "Convention and Limitation in Benefit-Cost Analysis”, Natural Resources Journal, vol. 6, núm. 2, 1966, pp. 195-222.

Harty, Harry P., "Status of PPBs in Local and State Governments in the United States", Policy Sciences, vol. 2, núm. 2, 1971, pp. 177-189.

Hird, John A., Policy Analysis in the States: Power, Knowledge, and Politics, Washington, D. C., Georgetown University Press, 2005.

Hitch, Charles, "Sub-Optimization in Operations Problems", Journal of the Operations Research Society of America, vol. 1, núm. 3, 1953, pp. 87-99.

Kaufman, Herbert, "Emerging Conflicts in the Doctrines of Public Administration", American Political Science Review, vol. 50, núm. 4, 1956, pp. $1057-1073$.

Kravitz, Walter, "The Advent of the Modern Congress: The Legislative Reorganization Act of 1970", Legislative Studies Quarterly, vol. 15, núm. 3, 1990, pp. 375-399.

Lee, Mordecai, Bureaus of Efficiency: Reforming Local Government in the Progressive Era, Milwaukee, Marquette University, 2008.

McGann, James G., Think Tanks and Policy Advice in the US: Think Tanks and Civil Societies Program, Filadelfia, University of Pennsylvania, 2005.

Medvetz, Thomas, Think Tanks in America, Chicago, University of Chicago Press, 2012.

Melkers, Julia y Katherine Willoughby, "Models of Performance-Measurement Use in Local Governments: Understanding Budgeting, Communication, and Lasting Effects", Public Administration Review, vol. 65, núm. 2, 2005, pp. 180-190. 
McCubbins, Mathew D., Roger G. Noll y Barry R. Weingast, "Administrative Procedures as Instruments of Political Control", Journal of Law, Economics and Organization, vol. 3, núm. 2, 1987, pp. 243-277.

Milward, H. Brinton y Keith G. Provan, "Governing the Hollow State", Journal of Public Administration Research and Theory, vol. 10, núm. 2, 2000, pp. 359-380.

Morgan, M. Granger y Jon M. Peha (eds.), Science and Technology Advice for Congress, Washington, D. C., Resources for the Future, 2003.

Mosher, Frederick C., A Tale of Two Agencies: A Comparative Analysis of the General Accounting Office and the Office of Management and Budget, Baton Rouge, Louisiana State University Press, 1984.

Nelson, Robert H., "The Office of Policy Analysis in the Department of the Interior", Journal of Policy Analysis and Management, vol. 8, núm. 3, 1989, pp. 395-410.

New York Bureau of Municipal Research, Government of the City of Rochester, N.Y.: General Survey, Critical Appraisal, and Constructive Suggestions. Prepared for the Rochester Bureau of Municipal Research, Albany, J. B. Lyon Company (Printers), 1915.

Office of Management and Budget, "Regulatory Analysis", OMB Circular A-4, Washington, D. C., Office of Management and Budget, 17 de septiembre de 2003.

Petracca, Mark, "Federal Advisory Committees, Interest Groups, and the Administrative State”, Congress Ẽ The Presidency, vol. 13, núm. 1, 1986, pp. 83-114.

Radin, Beryl, Beyond Machiavelli: Policy Analysis Reaches Midlife, $2^{\mathrm{a}}$ edición, Washington, D. C., Georgetown University Press, 2013.

Schacter, Hindy Lauer, "Philadelphia's Progressive-Era Bureau of Municipal Research", Administrative Theory Ẽ Praxis, vol. 24, núm. 3, 2002, pp. 555-570.

, "Democracy, Scientific Management and Urban Reform the Case of the Bureau of Municipal Research and the 1912 New York City School Inquiry", Journal of Management History, vol. 1, núm. 2, 1995, pp. 52-64.

Sherwin, Edward, "The Cost-Benefit Analysis of Financial Regulation: Lessons from the SEC's Stalled Mutual Fund Reform Effort”, Stanford Journal of Law, Business, and Finance, 12, 2006, pp. 1-60.

Shultz, George P., Memorandum for the Heads of Departments and Agencies, 
Washington, D. C., Office of Management and Budget, 5 de octubre de 1971.

Smith, Bruce L. R., The Advisors: Scientists in the Policy Process, Washington, D. C., Brookings Institution Press, 1992.

Stewart, Richard B., "The Reformation of Administrative Law", Harvard Law Review, vol. 88, núm. 8, 1975, pp. 1669-1813.

Teske, Paul, Regulation in the States, Washington, D. C., Brookings Institution Press, 2004.

Think Tanks and Civil Society Program, 2013 Global Go To Think Tank Index Report, Filadelfia, University of Pennsylvania, 22 de enero de 2014.

U.S. Congressional Research Service, Annual Report of the Congressional Research Service of the Library of Congress for Fiscal Year 2012, Washington, D. C., Congressional Research Service, 2013.

U.S. Government Accountability Office, Summary of GAO's Performance and Accountability Report: Fiscal Year 2014, GAO-14-3SP, 2014.

U.S. Government Accountability Office, Federal Advisory Committees: Issues Related to Independence and Balance of Advisory Committees, GAO-08-11T, 2008.

U.S. Government Services Administration, Federal Advisory Committee Act (FACA) Management Overview, http:/ / www.gsa.gov/portal/content/10 4514, 5 de enero 2015.

Vining, Aidan R. y David L. Weimer, "Assessing the Costs and Benefits of Social Policies”, en D. Weimer y A. Vining (eds.), Investing in the Disadvantaged: Assessing the Benefits and Costs of Social Programs, Washington, D. C., Georgetown University Press, 2009, pp. 1-16.

—, "Government Supply and Government Production Failure: A Framework Based on Contestability”, Journal of Public Policy, vol. 10, núm. 1, 1990, pp. 1-22.

—, Policy Analysis: Concepts and Practice, Nueva Jersey, Pearson, $5^{\mathrm{a}}$ edición, 2011.

Weaver, R. Kent, "The Changing World of Think Tanks", PS: Political Science Ẽ Politics, vol. 22, núm. 3, 1989, pp. 563-578.

Weimer, David L., Medical Governance: Values, Expertise, and Interests in Organ Transplantation, Washington, D. C., Georgetown University Press, 2010.

_ , "Stakeholder Governance of Organ Transplantation: A Desirable Model for Inducing Evidence-Based Medicine?", Regulation $\mathcal{E}^{\circ}$ Governance, vol. 4, núm. 3, 2010, pp. 281-302. 
— "The Puzzle of Private Rulemaking: Scientific Expertise, Flexibility, and Blame Avoidance in Regulation", Public Administration Review, vol. 66, núm. 4, 2006, pp. 569-582.

—, "Claiming Races, Broiler Contracts, Heresthetics, and Habits: Ten Concepts for Policy Design”, Policy Sciences, vol. 25, núm. 2, 1992, pp. 135-159.

— , "Institutionalizing Neutrally Competent Policy Analysis: Resources for Promoting Objectivity and Balance in Consolidating Democracies”, Policy Studies Journal, vol. 33, núm. 2, 2005, pp. 131-146.

Weimer, David L. y Aidan R. Vining, Policy Analysis: Concepts and Practice, $5^{\mathrm{a}}$ ed., Pearson, Upper Saddle River, NJ, 2011.

Wildavsky, Aaron, "Rescuing Policy Analysis from PPBS", Public Administration Review, vol. 29, núm. 2, 1969, pp. 189-202.

Wolf, Patrick J., "Neutral and Responsive Competence: The Bureau of the Budget, 1939-1948 Revisited”, Administration and Society, vol. 31, núm. 1, 1999, pp. 142-167. 